\title{
Out-of-equilibrium phonons in gated superconducting switches
}

\author{
M. F. Ritter', N. Crescini', D. Z. Haxell', M. Hinderling', H. Riel', C. Bruder'ㄹ, A. Fuhrer ${ }^{1 凶}{ }^{1}$ and \\ F. Nichele ${ }^{1}{ }^{1 凶}$
}

Recent experiments have suggested that superconductivity in metallic nanowires can be suppressed by the application of modest gate voltages. The source of this gate action has been debated and either attributed to an electric-field effect or to small leakage currents. Here we show that the suppression of superconductivity in titanium nitride nanowires on silicon substrates does not depend on the presence or absence of an electric field at the nanowire, but requires a current of high-energy electrons. The suppression is most efficient when electrons are injected into the nanowire, but similar results are obtained when electrons are passed between two remote electrodes. This is explained by the decay of high-energy electrons into phonons, which propagate through the substrate and affect superconductivity in the nanowire by generating quasiparticles. By studying the switching probability distribution of the nanowire, we also show that high-energy electron emission leads to a much broader phonon energy distribution compared with the case where superconductivity is suppressed by Joule heating near the nanowire.

$\mathrm{t}$ tis generally thought that metallic nanostructures are not affected by electric fields, as long as their size is larger than the corresponding screening length, which is typically below $1 \mathrm{~nm}$. Recent experiments ${ }^{1-6}$ have, however, shown that gate voltages can have a dramatic impact on the superconducting properties of metallic devices, including the ambipolar quenching of the critical current. The microscopic mechanism responsible for this behaviour has sparked debate. First, it was suggested that an electric field can penetrate a superconducting film up to the London penetration depth ${ }^{1}$. Second, it was proposed that an electric field might perturb the polarization of atomic orbitals at the metal surface, and this would affect the superconducting properties in the bulk ${ }^{7,8}$. Third, studies of the switching probability distribution (SPD) in metallic nanowires suggested an interplay between an electric field and superconducting phase slips 5 .

We have previously reproduced the most distinctive features of these experiments using titanium nitride (TiN), niobium and titanium nanowires 9 . In our samples, the critical current suppression was always accompanied by a current flowing between the gate and nanowire. In these experiments, the gate current is carried by electrons with energies of several electronvolts, which is orders of magnitude larger than the superconducting energy gap in the nanowires. We concluded that the emission of relatively few electrons leads to an avalanche of quasiparticles, which effectively quench the critical current ${ }^{10}$. This hypothesis was supported by tunnelling spectroscopy experiments ${ }^{11}$, which highlighted a non-thermal increase in quasiparticle population as a gate voltage was applied. Further work also demonstrated a correlation between the onset of gate currents and suppression of superconducting properties $^{12,13}$. However, open questions remain. For example, in a scenario where the injection of high-energy electrons controls the critical current suppression, a marked asymmetry would naively be expected between injecting high-energy electrons into the nanowire (negative gate voltage) and extracting electrons from the nanowire at the Fermi energy (positive gate voltage), as well as having them relax either in the substrate or in the gate electrode. Unravelling the microscopic mechanisms behind these observations could prove valuable in the development of technological applications of the phenomenon, such as the realization of voltage-controlled superconducting switches and resonators.

In this Article, we show that the quenching of superconductivity in metallic nanowires can be linked to the relaxation of high-energy electrons, and not to the presence of electric fields at the superconductor surface. In particular, we examine the effect of high-energy electrons flowing into the nanowire, out of the nanowire and between two remote gate electrodes in the vicinity of the nanowire. Detailed measurements reveal that superconductivity is most efficiently suppressed when a current is injected into the nanowire. However, a qualitatively similar critical current suppression is observed when high-energy electrons flow near the nanowire, without any current or electric field directly reaching the nanowire itself. The non-local nature of the observed effect is consistent with the energy relaxation of electrons by phonon emission in the substrate. Due to their relatively high energy, phonons generate quasiparticles in the superconductors and efficiently quench the critical current in our devices. At cryogenic temperatures, phonons can propagate over considerable distances in the crystalline silicon substrate before thermalizing. The effect is, thus, distinct from the situation where a local temperature increase is produced by a resistive heater. Our observations question the existing interpretations and theories based on electric fields, and provide an insight into the complex interactions between out-of-equilibrium phenomena in solids and the performance of superconducting hardware.

\section{Critical current suppression and electric fields}

Seven TiN nanowires on $\mathrm{Si}$ substrates were investigated during this work. All the nanowires had a length of $2 \mu \mathrm{m}$, width of $80 \mathrm{~nm}$ and height of $20 \mathrm{~nm}$. At low temperatures, the devices showed critical currents $I_{\mathrm{C}}$ between 42 and $45 \mu \mathrm{A}$, retrapping current $I_{\mathrm{R}}=1.0 \mu \mathrm{A}$ and normal-state resistance $R_{\mathrm{N}} \approx 1,750 \Omega$, consistent with a previous work ${ }^{9}$. The uniformity of these values demonstrates that the nanowires were homogeneous and not characterized by accidental weak

IIBM Quantum, IBM Research-Zurich, Rüschlikon, Switzerland. '2Department of Physics, University of Basel, Basel, Switzerland.

凶e-mail: afu@zurich.ibm.com; fni@zurich.ibm.com 
a

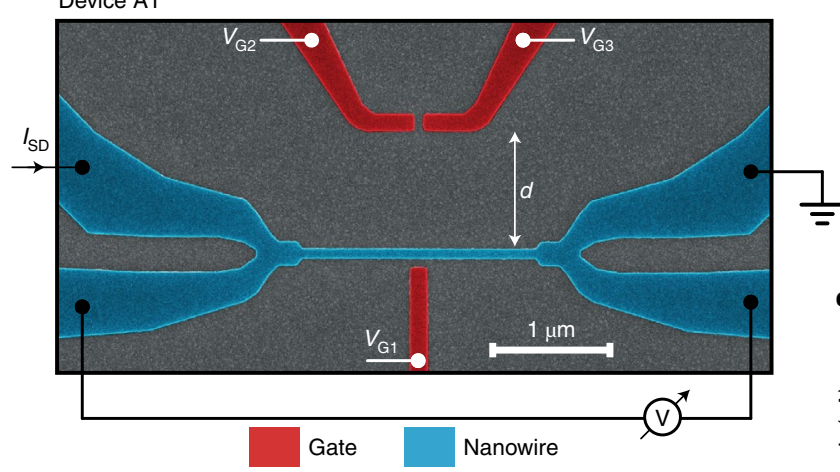

c

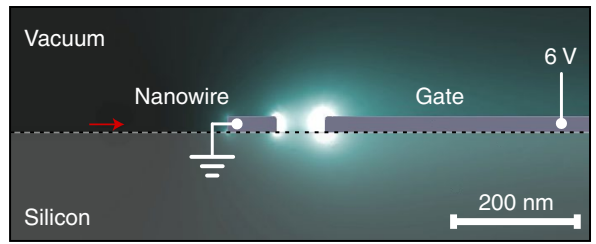

b

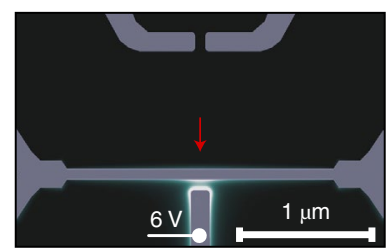

$\mathbf{f}$

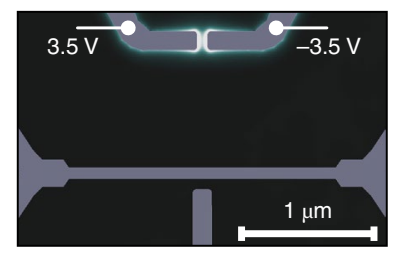

d
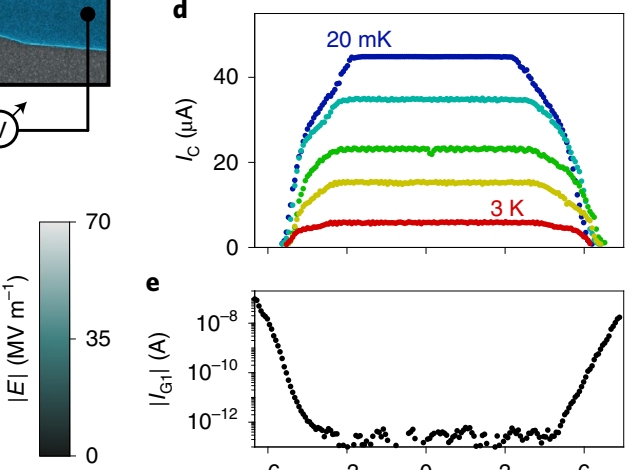

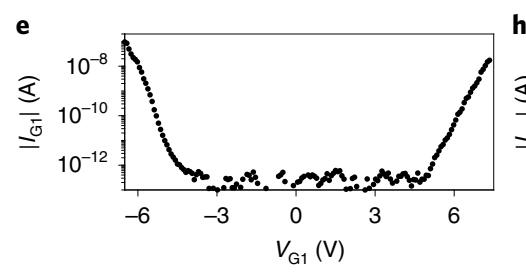

$h$ g
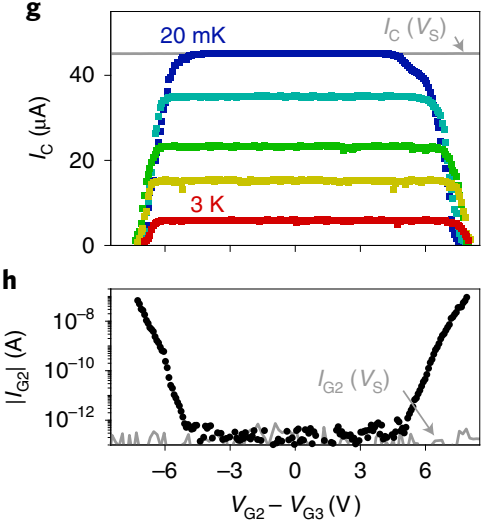

Fig. 1 | Basic device characterization and electric-field simulation. a, False-colour scanning electron micrograph of device A1, with a simplified measurement configuration. The nanowire under investigation is depicted in blue and the gates in red. $\mathbf{b}$, Finite element simulation of the electric-field magnitude $|E|$ for $V_{G 1}=6 \mathrm{~V}$. We show a slice of the 3D simulation on a plane elevated $10 \mathrm{~nm}$ from the Si substrate. $\mathbf{c}$, Same as in $\mathbf{b}$, but for a plane perpendicular to the substrate and intersecting gate 1 . The red arrow indicates the direction of the cut in $\mathbf{b}$. $\mathbf{d}$, Critical current $I_{\mathrm{C}}$ in device $\mathrm{A} 1$ as a function of gate voltage $V_{\mathrm{G} 1}$ for temperatures $T$ of $20 \mathrm{mK}$ (blue), $1.5 \mathrm{~K}, 2.1 \mathrm{~K}, 2.5 \mathrm{~K}$ and $3.0 \mathrm{~K}$ (red). e, Gate current $I_{\mathrm{G} 1}$ as a function of $V_{\mathrm{G} 1}$ measured at $T=20 \mathrm{mK}$ simultaneously to the data in $\mathbf{d}$. $\mathbf{f}$, Finite element simulation as in $\mathbf{b}$, but calculated for gate voltage difference $V_{\mathrm{G} 2}-V_{\mathrm{G} 3}=7 \mathrm{~V}$. $\mathbf{g}_{\text {, }} \mathrm{Critical}$ current $I_{\mathrm{C}}$ in device $A 1$ as a function of $V_{G 2}-V_{G 3}$ for temperatures as in $\mathbf{d}$ (markers), together with $I_{C}$ as a function of $V_{S}=2 V_{G 2}=2 V_{G 3}$, representing twice the voltage simultaneously applied to both gates (grey line). h, Current $I_{\mathrm{G} 2}$ flowing from gate 2 as a function of voltage difference $V_{\mathrm{G} 2}-V_{\mathrm{G} 3}$. In this configuration, $I_{\mathrm{G} 2}=-I_{\mathrm{G} 3}$ within the experimental error. Gate current $I_{\mathrm{G} 2}$ as a function of $V_{\mathrm{S}}$ is shown in grey.

links. The large difference between $I_{\mathrm{C}}$ and $I_{\mathrm{R}}$ indicates substantial self-heating in the normal state, together with limited heat extraction via the leads or the substrate, typical of metallic nanowires ${ }^{14,15}$. Further details on sample fabrication and basic characterization are reported in another study ${ }^{9}$ and in Methods. Here we present the results from four devices, referred to as devices A1, A2, B and C. Extended data and three additional devices, used as references, are shown in more detail in Supplementary Figs. 1-3.

Figure 1a shows a false-coloured scanning electron micrograph of device A1, together with the schematic of the measurement configuration. Device A1 consists of a nanowire (blue) and three gates (red). Gate 1, controlled by voltage $V_{\mathrm{G} 1}$, was separated from the nanowire by a gap of $80 \mathrm{~nm}$. Gates 2 and 3, controlled by voltages $V_{\mathrm{G} 2}$ and $V_{\mathrm{G} 3}$, respectively, were separated from each other by $80 \mathrm{~nm}$ and from the nanowire by a distance $d=1 \mu \mathrm{m}$. A similar device, named device A2, had $d=80 \mathrm{~nm}$ (Supplementary Fig. 1).

We first discuss the response of device A1 to a side-gate voltage $V_{\mathrm{G} 1}$, similar to previous work ${ }^{1,9,11}$. The electric-field distribution in this configuration was calculated using three-dimensional (3D) finite element simulations (Methods). Figure 1b shows the field magnitude $|E|$ on a plane $10 \mathrm{~nm}$ above the substrate for $V_{\mathrm{G} 1}=6 \mathrm{~V}$. Figure $1 \mathrm{c}$ represents $|E|$ on a plane perpendicular to both substrate and wire axis, and intersecting the gate (Fig. 1b, red arrow). To better highlight the field distribution, the colour scale was saturated to $|E|=70 \mathrm{MV} \mathrm{m}^{-1}$. The highest $|E|$ in our simulations was below $|E|=500 \mathrm{MV} \mathrm{m}^{-1}$, which is several orders of magnitude smaller than typical electric fields required to perturb superconductivity in a metallic device ${ }^{16-18}$. Figure $1 \mathrm{~d}$ shows the experimentally measured $I_{\mathrm{C}}$ as a function of $V_{\mathrm{G} 1}$, for temperatures ranging from $20 \mathrm{mK}$ (blue) to $3 \mathrm{~K}$ (red). Figure 1e shows the gate current $I_{\mathrm{G} 1}$ simultaneously measured to the data in Fig. 1d. Consistent with previous observations ${ }^{9,12}$, the decrease in $I_{\mathrm{C}}$ was correlated to the onset of $I_{\mathrm{G} 1}$, and the initial decrease in $I_{\mathrm{C}}$ took place for $I_{\mathrm{G} 1}<1 \mathrm{pA}$. Furthermore, $\left|I_{\mathrm{G} 1}\right|$ was found to exponentially increase with $V_{\mathrm{G} 1}$ and to be approximately symmetric around $V_{\mathrm{G} 1}=0$.

We now discuss the dependence of $I_{\mathrm{C}}$ on a differentially applied voltage $V_{\mathrm{G} 2}-V_{\mathrm{G} 3}$, with $V_{\mathrm{G} 2}=-V_{\mathrm{G} 3}$. Figure 1f shows the numerically computed electric field for $V_{\mathrm{G} 2}-V_{\mathrm{G} 3}=7 \mathrm{~V}$. As expected, $|E|$ is strongly confined between gates 2 and 3 . If superconductivity in the nanowire were controlled by the electric fields, this configuration should result in negligible effects on $I_{\mathrm{C}}$. Strikingly, quenching of the supercurrent occurred even in this situation (Fig. 1g). Figure $1 \mathrm{~h}$ shows the current $I_{\mathrm{G} 2}$ flowing from gate 2 (we found that $I_{\mathrm{G} 2}=-I_{\mathrm{G} 3}$ within the experimental error). Remarkably, the suppression of $I_{\mathrm{C}}$ was strongly correlated to the onset of $I_{\mathrm{G} 2}$, despite no measurable gate current reaching the nanowire and negligible electric fields between the gate and nanowire.

To test whether residual electric fields were relevant, we also measured $I_{\mathrm{C}}$ with gates 2 and 3 biased at the same voltage $\left(V_{\mathrm{G} 2}=V_{\mathrm{G} 3}\right)$. In Fig. $1 \mathrm{~g}$, we plot $I_{\mathrm{C}}$ as a function of the quantity $V_{\mathrm{S}}=2 V_{\mathrm{G} 2}=2 V_{\mathrm{G} 3}$ (Fig. $1 \mathrm{~g}$, solid grey line) as, at any one point in this plot, the absolute voltages $\left|V_{\mathrm{G} 2}\right|$ and $\left|V_{\mathrm{G} 3}\right|$ on the gate electrodes are identical and the absolute value of the electric field $|E|$ reaching the nanowire is similar. More specifically, we estimate $\left|E\left(V_{\mathrm{G} 2}=V_{\mathrm{G} 3}\right)\right| \gtrsim\left|E\left(V_{\mathrm{G} 2}=-V_{\mathrm{G} 3}\right)\right|$ at the nanowire surface. Nevertheless, no current was detected between the gates and nanowire for symmetrically applied gate voltages (Fig. $1 \mathrm{~h}$, grey curve) and $I_{\mathrm{C}}$ was not perturbed. These results further corroborate our findings that high-energy electrons, and not electric fields, are responsible for the suppression of $I_{\mathrm{C}}$. Similar results obtained with device A2 are presented in Supplementary Fig. 1.

Overall, experiments and numerical simulation presented in Fig. 1 demonstrate that the suppression of superconductivity takes place 

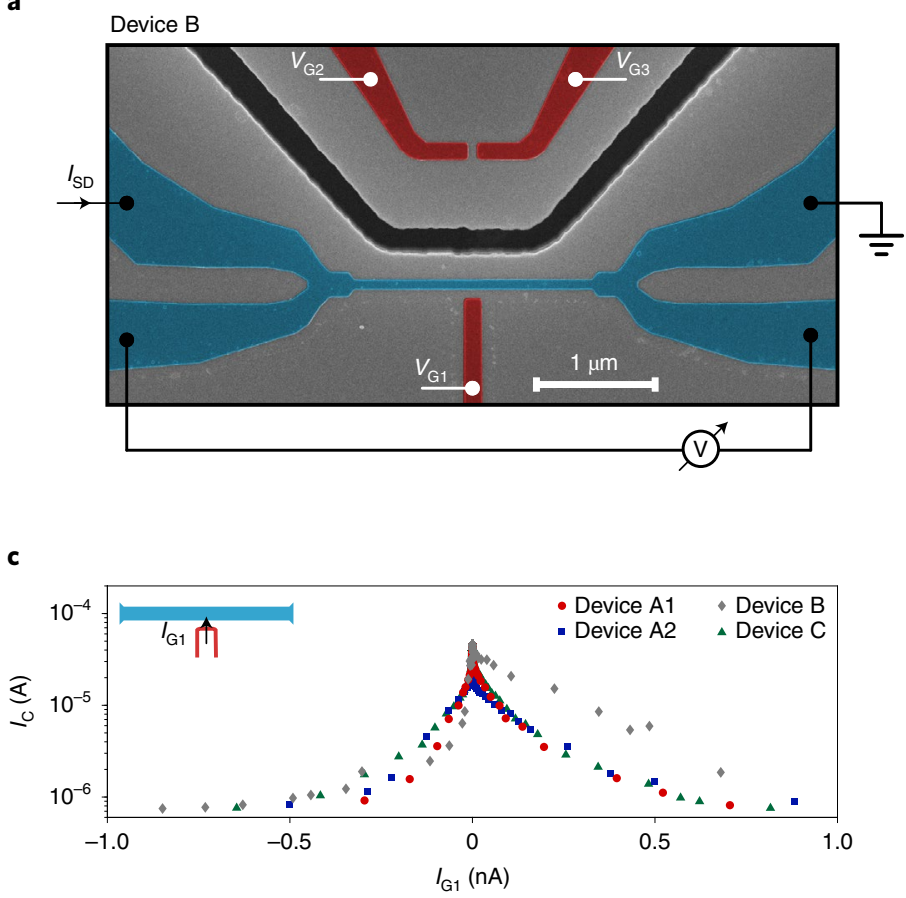

d

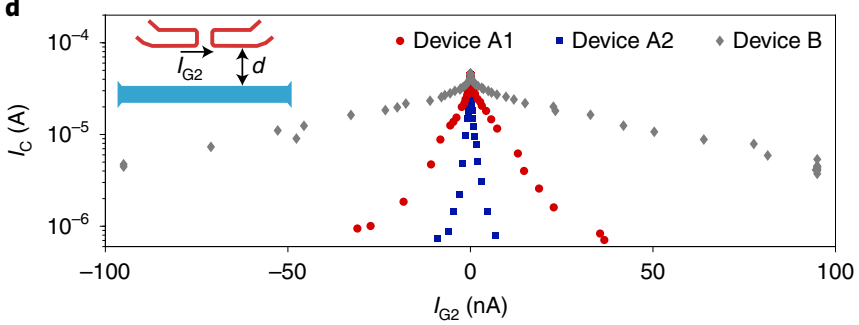

b

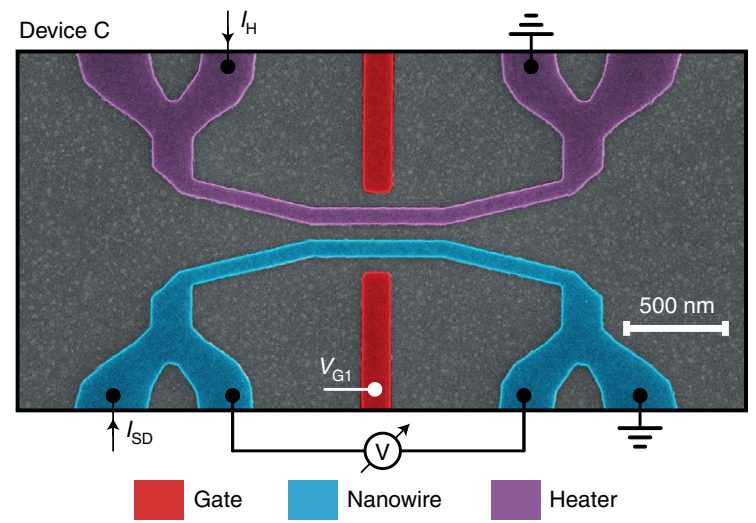

e

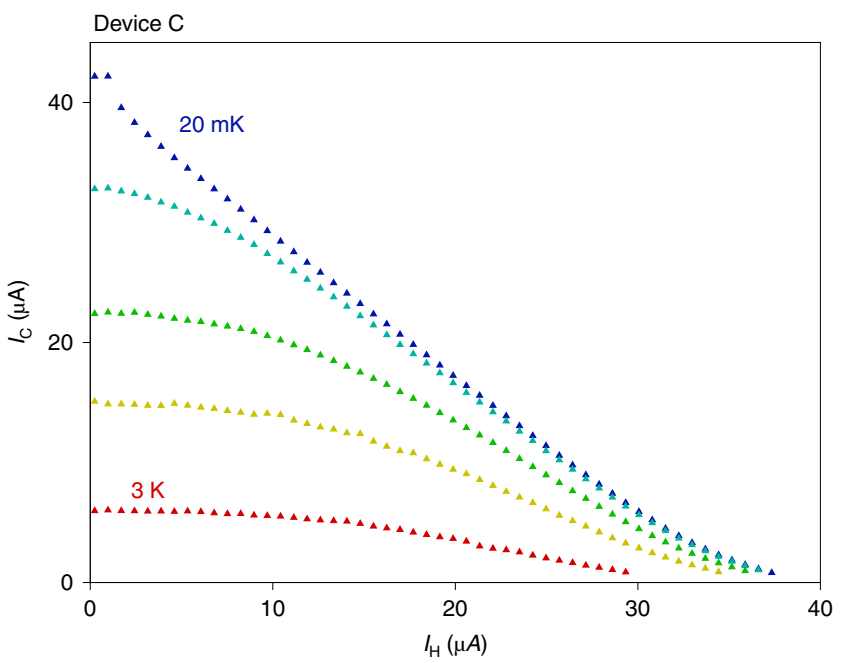

Fig. 2 | Additional devices. a, False-colour scanning electron micrograph of device B. The nanowire is depicted in blue; the gates, red; and the trench, black. The trench is $510 \mathrm{~nm}$ deep, $200 \mathrm{~nm}$ wide and has a total length of $80 \mu \mathrm{m}$. b. False-colour scanning electron micrograph of device $\mathrm{C}$. The nanowire under investigation is depicted in blue; the gates, red; and the heater nanowire, purple. c, Plot of critical current $I_{C}$ as a function of gate current $I_{\mathrm{G} 1}$ for all the devices presented in the main text. $\mathbf{d}$, Plot of $I_{C}$ as a function of the remote gate current $I_{G 2}$ for devices $A 1(d=1 \mu \mathrm{m}), A 2(d=80 \mathrm{~nm})$ and $B(d=1 \mu \mathrm{m}$ plus a trench). e, Critical current in device $C$ as a function of heater current $I_{\mathrm{H}}$ for temperatures as in Fig. $1 \mathrm{~d}$.

irrespective of the electric fields at the nanowire surface. Instead it requires the flow of high-energy electrons in the surroundings of the device. This is the first conclusion of our work.

\section{Role of substrate}

The remote action of $V_{\mathrm{G} 2}-V_{\mathrm{G} 3}$ on $I_{\mathrm{C}}$ points to the existence of an efficient energy transfer mechanism triggered by the flow of $I_{\mathrm{G} 2}$. We now analyse the origin of this remote action more carefully using devices B and C (Fig. 2a,b, respectively). Device B is identical to device A1, except for the presence of a 510-nm-deep, 200 -nm-wide, $80-\mu \mathrm{m}$-long trench etched into the substrate between the remote gates and nanowire. Device $\mathrm{C}$ consists of two parallel TiN nanowires separated by a distance of $80 \mathrm{~nm}$. Each nanowire was controlled by a nearby gate (Fig. $2 \mathrm{~b}$, red). We measured the critical current of one of the two nanowires (Fig. 2b, blue), whereas the second one (Fig. 2b, purple) was set in the resistive state and was traversed by a d.c. current $I_{\mathrm{H}}$, resulting in Joule heating, similar to another work ${ }^{19}$.

Figure 2c,d summarizes the behaviour of our devices in terms of $I_{\mathrm{C}}$ as a function of $I_{\mathrm{G} 1}$ and $I_{\mathrm{G} 2}$, respectively. The full dataset is presented in Supplementary Figs. 1 and 2. The dependence on $I_{\mathrm{G} 1}$ (Fig. $2 c)$ is similar in all the devices, with a faster suppression of $I_{C}$ for $I_{\mathrm{G} 1}<0$. Due to the exponential dependence of $I_{\mathrm{G} 1}$ on $V_{\mathrm{G} 1}$, this asymmetry is hard to spot in Figs. 1d,e. We further notice that device B (grey diamonds) exhibited a particularly slow decay of $I_{\mathrm{C}}$ for $I_{\mathrm{G} 1}>0$. We will discuss the possible causes for this asymmetry below. Figure $2 \mathrm{~d}$ reveals that $I_{\mathrm{G} 2}$ is considerably less effective in suppressing $I_{\mathrm{C}}$ than $I_{\mathrm{G} 1}$; furthermore, device A2 (blue squares; $d=80 \mathrm{~nm}$ ) was six times more efficient than device A1 (red circles; $d=1 \mu \mathrm{m}$ ), which was six times more efficient than device B (grey diamonds; $d=1 \mu \mathrm{m}$ plus an etched trench). In the case of device B, the maximum $I_{\mathrm{G} 2}$ allowed in our setup $(100 \mathrm{nA})$ was not sufficient to reach $I_{\mathrm{C}}=0$. Altogether, these results demonstrate that most of the remote action of $I_{\mathrm{G} 2}$ on $I_{\mathrm{C}}$ is mediated by the substrate, that is, the high-energy electrons relax by emitting phonons, which travel through the substrate and affect superconductivity in the nanowire. This is the second main conclusion of our work.

\section{Comparison to Joule heating}

We now discuss the properties of the generated phonons in more detail. In particular, we compare their effect on $I_{C}$ with that of the heat generated by a resistive conductor placed $80 \mathrm{~nm}$ from the superconducting nanowire. These experiments were performed with device C (Fig. 2b). The dependence of $I_{\mathrm{C}}$ on heater current $I_{\mathrm{H}}$ is shown in Fig. 2e for various temperatures. As expected, Joule heating eventually resulted in the suppression of $I_{\mathrm{C}}$. However, the current required to reach $I_{\mathrm{C}}=0$ was several orders of magnitude higher than in the configurations where a gate voltage was applied. 


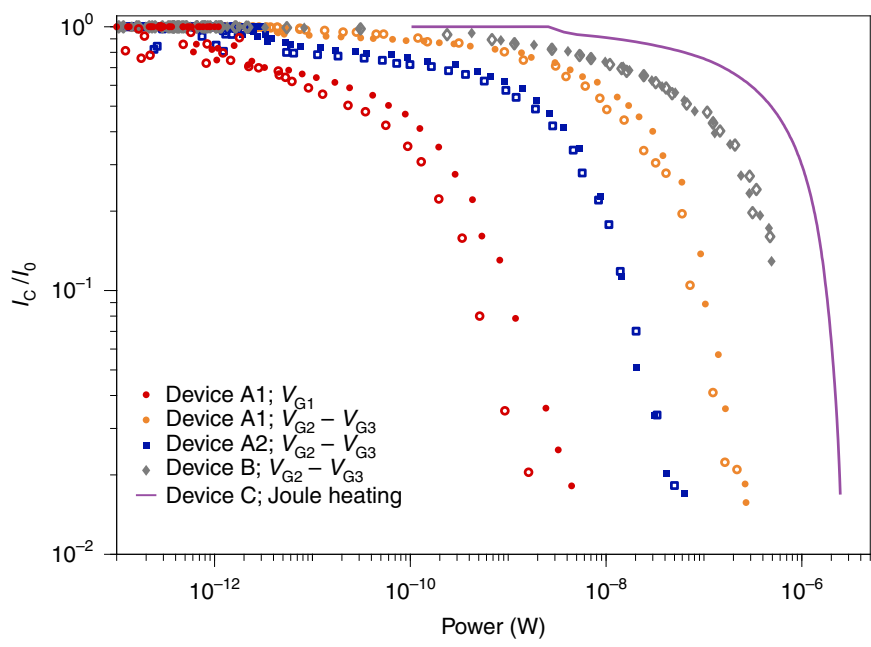

Fig. 3 | Comparison of switching power. Normalized critical current $I_{C} / I_{0}$ as a function of input power for various devices and experimental configurations. Full and empty markers define the positive and negative gate polarity, respectively. The solid purple line indicates the dependence as a function of Joule heating in device $\mathrm{C}$.

Figure 3 provides a comparison between the devices presented above in terms of the suppression of normalized critical currents $I_{C}$ as a function of dissipated power. For each measurement configuration, we distinguished the case of a positive and negative voltage bias with full and empty markers, respectively. Curves at higher temperatures are shown in Supplementary Fig. 4. The critical current is more efficiently suppressed when voltage bias $V_{\mathrm{G} 1}$ is applied to a gate directly facing the nanowire (red dots). In this case, the dissipated power is calculated as $I_{\mathrm{G} 1} V_{\mathrm{G} 1}$. When a remote current $I_{\mathrm{G} 2}$ flows, the power is calculated as $I_{\mathrm{G} 2}\left(V_{\mathrm{G} 2}-V_{\mathrm{G} 3}\right)$. Suppressing $I_{\mathrm{C}}$ by means of Joule heating with a resistive conductor (Fig. 3, purple line) required a considerably higher power $I_{\mathrm{H}}^{2} R_{\mathrm{N}}$ than the other configurations. As noted above, the dependence on $I_{\mathrm{G} 1}$ (Fig. 3, red circles) shows a difference between the positive and negative gate polarity, with the negative polarity being 2.5 times more power efficient in suppressing $I_{\mathrm{C}}$ compared with the positive one.

We have shown that Joule heating is orders of magnitude less efficient in suppressing $I_{C}$ of our nanowires than a current of high-energy electrons. In addition to these quantitative differences, we gain a further insight from the SPDs of our devices. The SPD is the probability of switching from the superconducting to resistive state to occur per unit of source-drain current. The SPD has proven to be a powerful tool to study Josephson junction and metallic nanowire properties that are hard to access with standard transport measurements $\mathrm{s}^{5,20}$. Figure $4 \mathrm{a}, \mathrm{b}$ shows the SPDs of devices A1 and $\mathrm{C}$, respectively, under various experimental conditions. For these experiments, the source-drain current was swept 20,000 times from 0 to $49 \mu \mathrm{A}$. For each sweep, the source-drain current value at which a switch to the resistive state occurred was recorded. At low temperature and zero gate voltage, device Al exhibited a sharp SPD (Fig. $4 \mathrm{a}$, blue markers), with a standard deviation $\sigma_{I}=47 \mathrm{nA}$. At a temperature of $2.2 \mathrm{~K}$ (Fig. $4 \mathrm{a}$, green markers), the SPDs had their maximum at half of the low-temperature $I_{\mathrm{C}}$ value, with $\sigma_{I}=100 \mathrm{nA}$. A more detailed analysis (Supplementary Fig. 5) revealed that the switching mechanisms at $20 \mathrm{mK}$ and $2.2 \mathrm{~K}$ are consistent with quantum phase slips and thermal fluctuations, respectively. Much broader SPDs were obtained by applying a gate leakage current $I_{\mathrm{G} 1}=10 \mathrm{pA}$ (Fig. $4 \mathrm{a}$, red markers), with $\sigma_{I}=2.0 \mu \mathrm{A}$. The finding that the application of a gate voltage results in much broader SPDs than increasing the bath temperature (for equal suppression of $I_{\mathrm{C}}$ ) is consistent with the observations elsewhere ${ }^{5}$. However, we show that a similarly broad
SPD is also obtained by applying a remote current $I_{\mathrm{G} 2}=2.5 \mathrm{nA}$ (Fig. $4 \mathrm{a}$, orange circles; $\sigma_{I}=1.2 \mu \mathrm{A}$ ), which is without any electric field or current reaching the nanowire. Using device $\mathrm{C}$ (Fig. 4b), we compare the SPD obtained when $I_{\mathrm{C}}$ is suppressed by $50 \%$ either by Joule heating (solid purple line) or by increasing the bath temperature to $2.1 \mathrm{~K}$ (green triangles). The two results are indistinguishable, indicating that a resistive heater indeed affects the superconductivity in the same way as an increase in the bath temperature, but in a totally different manner than a current of high-energy electrons (grey triangles). Because of the difference between the SPDs obtained at a high temperature (green markers) and finite gate voltage (red markers), another study ${ }^{5}$ excluded the presence of electrical currents. This conclusion was, however, reached under the assumption that a gate current causes heating similar to an increase in the bath temperature. Our results demonstrate, instead, that a current of high-energy electrons perturbs the superconducting properties of nanowires in a way that is qualitatively and quantitatively distinct from a bare temperature increase, even if the current does not flow into the nanowire but only in its surroundings. This is the third main conclusion of our work.

\section{Nature of generated phonons}

Our observations are consistent with the phenomenology of phonon generation by hot electrons in the substrate. First, we note that phonons with energies above the superconducting gap $(500 \mu \mathrm{eV}$ for TiN (ref. $\left.{ }^{21}\right)$ ) are well known to affect superconducting devices ${ }^{22-25}$. Second, electrons accelerated by high electric fields in Si undergo a series of relaxation events over timescales below 1 ns and on mean free paths below $10 \mathrm{~nm}$. Such relaxation most probably happens by the emission of optical and acoustic phonons ${ }^{26-29}$. Phonons in $\mathrm{Si}$ have a maximum energy of the order of $50 \mathrm{meV}$, which means that a single electron with an energy of a few electronvolts can generate a large amount of phonons ${ }^{30,31}$ as it travels between two metallic electrodes. At temperatures below $3 \mathrm{~K}$, phonons in Si have long mean free paths (up to $1 \mu \mathrm{m}$ (refs. ${ }^{32,33}$ )) and even longer thermalization lengths. It is, therefore, expected that the emitted phonons reach the nanowire in an out-of-equilibrium state ${ }^{34,35}$.

The electronic mean free path in Si decreases as $|E|$ increases $^{26,27}$, resulting in intense phonon emission close to the metal electrodes, independent of the gate voltage polarity (Fig. 1c) ${ }^{30}$. This may be the reason for the more efficient suppression of $I_{\mathrm{C}}$ when a current is either injected or extracted from the nanowire (Fig. 2c) compared with the case where a current flows between two gates near the nanowire (Fig. 2d, device A2). Furthermore, the suppression of $I_{\mathrm{C}}$ by a fixed factor requires 2.5 times less power for $V_{\mathrm{G} 1}<0$ (Fig. 3) compared with $V_{\mathrm{G} 1}>0$. This could indicate that electrons reaching the nanowire are not completely thermalized, and can still generate a sizeable number of quasiparticles via electron-electron interactions in the nanowire ${ }^{10,36,37}$. Assuming the phononic contribution is similar for both gate polarities (that is, phonon emission is isotropic), we estimate that more than half the suppression of $I_{\mathrm{C}}$ for $V_{\mathrm{G} 1}<0$ is due to electron-electron interaction. Future work might use more complex geometries to map out angular anisotropies in the phonon emission and absorption processes.

The broadening of SPDs with gate voltage is consistent with the nanowire being subject to extremely energetic events, capable of suppressing superconductivity even at small source-drain currents. The characteristic energy spread of such events can be quantified by the Kurkijavi power law $^{20}$, which allows one to relate the width of the SPD to the effective energy $E_{\text {eff }}$ (Supplementary Section 5). As shown by the red dots in Fig. 4 a, we obtain $E_{\text {eff }} \approx 8.6 \mathrm{meV}$, consistent with the idea that the energy of a leakage electron $(7 \mathrm{eV})$ dissipates in successive scattering events in the substrate before reaching the nanowire. In the case of a remote current (Fig. $4 \mathrm{a}$, orange dots), we get $E_{\text {eff }} \approx 6.3 \mathrm{meV}$, indicating that, on average, the phonons thermalize more over the longer distance. A possible framework for 

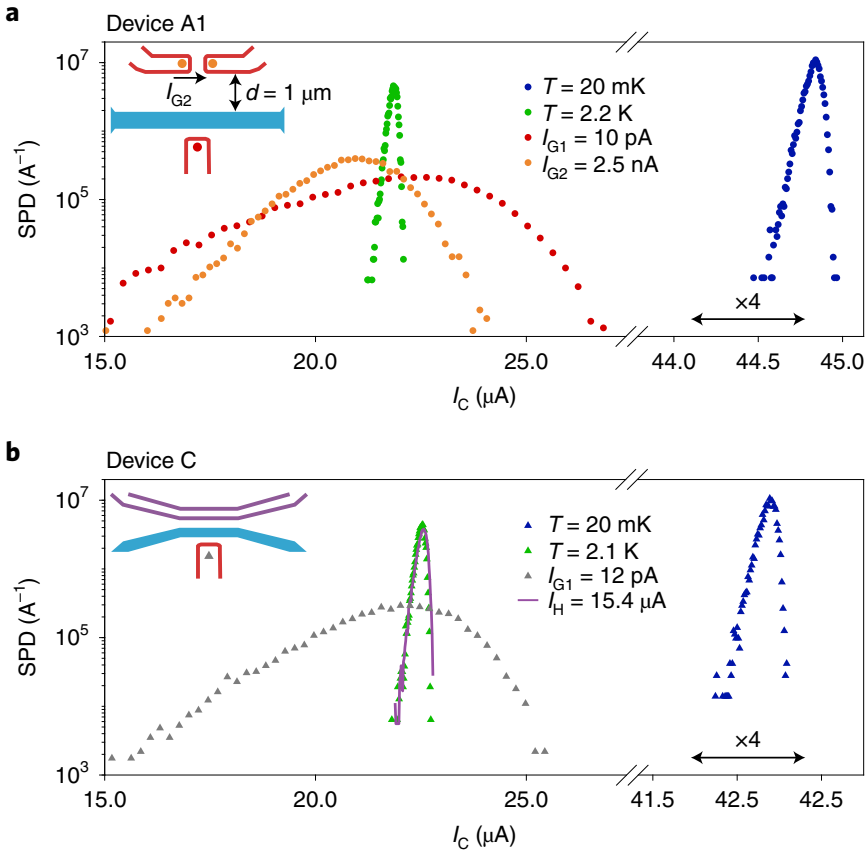

Fig. 4 | Comparison of SPDs. a, SPD in device A1 as a function of sourcedrain current $I_{\mathrm{SD}}$. The blue and green circles are obtained at zero gate voltage and for $T=20 \mathrm{mK}$ and $T=2.2 \mathrm{~K}$, respectively. The red circles are obtained for gate current $I_{\mathrm{G} 1}=10 \mathrm{pA}\left(V_{\mathrm{G} 1}=5.85 \mathrm{~V}\right)$ and the orange dots, for remote gate current $I_{G 2}=2.5 \mathrm{nA}\left(V_{G 2}-V_{G 3}=7.25 \mathrm{~V}\right)$. Note that the horizontal axis is interrupted and the high-current region is horizontally expanded by a factor of four. $\mathbf{b}$, Same data as in $\mathbf{a}$, but for device $\mathbf{C}$.

The blue and green triangles are obtained at zero gate voltage and for $T=20 \mathrm{mK}$ and $T=2.1 \mathrm{~K}$, respectively. The grey triangles are obtained for $I_{\mathrm{G} 1}=12 \mathrm{pA}\left(\mathrm{V}_{\mathrm{G} 1}=5.2 \mathrm{~V}\right)$ and the purple line is obtained for a heater current $I_{H}=15.4 \mu \mathrm{A}$.

analysing such SPDs in Josephson junctions subject to high-energy electrons was recently proposed elsewhere ${ }^{38}$.

In device $\mathrm{B}$, we noticed an anomalously large asymmetry in the parametric plot of $I_{\mathrm{C}}$ versus $I_{\mathrm{G} 1}$ (Fig. 2c). With three reference devices (Supplementary Fig. 3a), we confirmed that such an asymmetry is a robust feature that arises following the fabrication steps required to etch trenches into the substrate (Methods). Similarly, the efficiency of the remote action of $I_{\mathrm{G} 2}$ slightly decreased after additional fabrication, even when trenches were not etched (Supplementary Fig. 3b). Interestingly, no other sample parameters were affected by the additional fabrication steps. These results suggest that some of the out-of-equilibrium processes taking place in our device are sensitive to the surface treatment of the samples. Measuring device $B$, we have shown that out-of-equilibrium phonons are primarily responsible for the remote action of $I_{\mathrm{G} 2}$ on $I_{\mathrm{C}}$. However, our work does not exclude the presence of additional energy relaxation mechanisms that contribute, together with phonons, to the suppression of $I_{\mathrm{C}}$ (such as photon emission). Previous works detected photons in a variety of devices as a result of tunnelling events ${ }^{39-43}$ as well as bremsstrahlung and carrier recombination of high-energy electrons ${ }^{44,45}$. It is also well known that superconducting nanowires $^{46}$ and Josephson junctions ${ }^{47}$ are highly sensitive to the impact of high-energy photons. Both phonon and photon transport may be affected by the additional fabrication steps for trenching, for example, by a change in surface roughness or dielectric properties. The relative contribution of phonons and photons is estimated by comparing the response of devices $\mathrm{A} 1$ and $\mathrm{B}$ to $V_{\mathrm{G} 2}-V_{\mathrm{G} 3}$ (Fig. 3). Device B required a six times higher power to reach the same $I_{\mathrm{C}} / I_{0}$, indicating that the trench blocks five-sixth of the power that would have been otherwise absorbed by the nanowire. If we assume that any photonic contributions are unaffected by the trench, we can calculate an upper bound on such a contribution in that it must be smaller than one-fifth of the phononic contribution. Note that the reduced power reaching the nanowire in device $B$ could also be carried by phonons, which-if travelling deep in the substrate-are also not affected by the trench.

\section{Conclusions}

We have reported a comprehensive study of the mechanism responsible for the suppression of critical currents in metallic nanowires in the presence of large gate voltages. We have shown that previously reported features, which were attributed to the electric field on the superconductor, can be obtained in the absence of electric fields. Our data indicate that critical currents are suppressed as a consequence of the relaxation of high-energy electrons, either in the substrate or in the electrodes. Our results also elucidate the mechanism behind the ambipolar suppression of $I_{\mathrm{C}}$ as a function of gate voltage (Fig. 1d), which was not fully explained in previous works ${ }^{9,11,12}$. The ambipolar suppression of $I_{\mathrm{C}}$ requires an approximately symmetric gate current (which is experimentally observed (Fig. 1e)) as well as an efficient energy equilibration mechanism between the gate and nanowire. Energy equilibration is dominated in our devices by energetic phonons spreading through the substrate over distances in excess of $1 \mu \mathrm{m}$. Although this remote action may pose a limit to the device integration density, it could also open new paths for device design. For example, it could be used to develop efficient superconducting switches ${ }^{48-51}$ that do not require the injection of electrons into the switching element, but are instead mediated by high-energy phonons that are guided towards a switching element. It also opens new possibilities to investigate the interplay between out-of-equilibrium phenomena, resulting quasiparticle generation and superconducting quantum hardware.

\section{Methods}

Sample fabrication. A 20 -nm-thick TiN film was sputtered on a Si substrate. The Si substrate used for this work was intrinsic and became insulating at temperatures below $100 \mathrm{~K}$. Before TiN deposition, the Si chip was immersed in a buffered hydrofluoric acid (HF) solution for the removal of native oxides. The TiN film showed a critical temperature of $3.7 \mathrm{~K}$ and a resistivity of $68 \Omega \mathrm{sq}^{-1}$. Devices were defined by electron-beam lithography on a negative hydrogen silsesquioxane resist and dry etching in $\mathrm{HBr}$ plasma. The resist was then removed by immersion in HF. The devices were contacted by Ti/Au bond pads defined by optical lithography and metal evaporation. Some devices were further processed after the deposition of bond pads. In this case, $2 \mathrm{~nm} \mathrm{Si}_{3} \mathrm{~N}_{4}$ and $210 \mathrm{~nm} \mathrm{SiO} \mathrm{S}_{2}$ hard mask were deposited by atomic layer deposition and plasma-enhanced chemical vapour deposition, respectively. A trench was defined in the hard mask with electron-beam lithography and CSAR AR-P 6200.09 resist, standard development and reactive ion etching of the $\mathrm{SiO}_{2}$ layer. The $\mathrm{Si}$ substrate was further etched in inductively coupled $\mathrm{HBr}$ plasma. Finally, the hard mask was etched in buffered HF.

Electrical measurements. Measurements were performed in a dilution refrigerator with a base temperature of $20 \mathrm{mK}$. Critical currents $I_{\mathrm{C}}$ were measured by applying a sawtooth wave $I_{\mathrm{SD}}$ signal with an amplitude of $49 \mu \mathrm{A}$ and repetition rates between 33 and $133 \mathrm{~Hz}$, whereas voltages $V$ across the nanowires were recorded by a digital oscilloscope. The measurement setup was synchronized so that a switch from zero to a finite voltage in the oscilloscope could be related to the source-drain current at which the switch occurred. This technique allowed us to reliably extract critical currents down to $700 \mathrm{nA}$. The critical currents presented in Fig. 1 were obtained by averaging 108 such switching events. Sporadic fluctuations of $I_{\mathrm{C}}$ visible at $T \geq 1.5 \mathrm{~K}$ are associated with the instabilities of the temperature controller. The SPDs presented in Fig. 4 were obtained by recording 20,000 switches over a time interval of $10 \mathrm{~min}$. To keep the nanowire potential constant as $I_{\mathrm{SD}}$ varies, $I_{\mathrm{SD}}$ was generated by sourcing two synchronized sawtooth waves with opposite polarities into $163 \mathrm{k} \Omega$ resistors placed at both ends of the nanowire (which add to the existing line resistance of $2.2 \mathrm{k} \Omega$ ). Gate voltages were applied via high-precision source-measure units, which recorded the current flowing into the gate contacts. The gate current data shown in Fig. 1e,h were obtained after subtracting the linear components ranging between 1 and $5 \mathrm{pA} \mathrm{V}^{-1}$, as discussed elsewhere ${ }^{9}$. Such resistive contributions are attributed to spurious leakage paths in our setup. 
Electrostatic simulations. The electric-field distributions presented in Fig. 1 were produced with finite-element 3D electrostatic simulations performed with Ansys Maxwell version 2019R2. A substrate permittivity of 12 was assumed to resemble the electromagnetic properties of silicon, and its thickness was set to $1 \mu \mathrm{m}$. The metallic layer comprising the nanowire and gate electrodes was modelled as a 20 -nm-thick perfect conductor. The upper edges of the structures were filleted with a radius of $3 \mathrm{~nm}$. The geometry of the nanowire and gates was generated from the same layout file used for the electron-beam lithography of the devices. The fields shown in Fig. 1b, f are slices of the 3D simulation taken at half the height of the nanowire. Figure $1 \mathrm{c}$ shows the image taken perpendicular to the substrate and intersecting the gate electrode. The colour scale was saturated to a maximum value of $70 \mathrm{MV} \mathrm{m}^{-1}$ to evidence the field distribution, whereas the full scale reached up to $500 \mathrm{MV} \mathrm{m}^{-1}$

\section{Data availability}

The data presented in this work are available at https://doi.org/10.5281/ zenodo.5825804. Further data that support the findings of this study are available from the corresponding authors upon reasonable request.

Received: 3 June 2021; Accepted: 19 January 2022;

Published online: 28 February 2022

\section{References}

1. De Simoni, G., Paolucci, F., Solinas, P., Strambini, E. \& Giazotto, F. Metallic supercurrent field-effect transistor. Nat. Nanotechnol. 13, 802-805 (2018).

2. Paolucci, F., De Simoni, G., Strambini, E., Solinas, P. \& Giazotto, F. Ultra-efficient superconducting Dayem bridge field-effect transistor. Nano Lett. 18, 4195-4199 (2018).

3. De Simoni, G., Paolucci, F., Puglia, C. \& Giazotto, F. Josephson field-effect transistors based on all-metallic $\mathrm{Al} / \mathrm{Cu} / \mathrm{Al}$ proximity nanojunctions. ACS Nano 13, 7871-7876 (2019).

4. Paolucci, F. et al. Field-effect controllable metallic Josephson interferometer. Nano Lett. 19, 6263-6269 (2019).

5. Puglia, C., De Simoni, G. \& Giazotto, F. Electrostatic control of phase slips in Ti Josephson nanotransistors. Phys. Rev. Appl. 13, 054026 (2020).

6. Rocci, M. et al. Gate-controlled suspended titanium nanobridge supercurrent transistor. ACS Nano 14, 12621-12628 (2020).

7. Mercaldo, M. T., Solinas, P., Giazotto, F. \& Cuoco, M. Electrically tunable superconductivity through surface orbital polarization. Phys. Rev. Appl. 14, 034041 (2020).

8. Paolucci, F. et al. Magnetotransport experiments on fully metallic superconducting Dayem-bridge field-effect transistors. Phys. Rev. Appl. 11, 024061 (2019).

9. Ritter, M. F. et al. A superconducting switch actuated by injection of high-energy electrons. Nat. Commun. 12, 1266 (2021).

10. Engel, A. \& Schilling, A. Numerical analysis of detection-mechanism models of superconducting nanowire single-photon detector. J. Appl. Phys. 114 214501 (2013)

11. Alegria, L. D. et al. High-energy quasiparticle injection into mesoscopic superconductors. Nat. Nanotechnol. 16, 404-408 (2021).

12. Golokolenov, I., Guthrie, A., Kafanov, S., Pashkin, Y. A. \& Tsepelin, V. On the origin of the controversial electrostatic field effect in superconductors. Nat. Commun. 12, 2747 (2021).

13. Catto, G., Liu, W., Kundu, S., Lahtinen, V. \& Möttönen, M. Microwave response of a metallic superconductor subject to a high-voltage gate electrode. Preprint at https://arxiv.org/abs/2105.08322 (2021).

14. Tinkham, M., Free, J. U., Lau, C. N. \& Markovic, N. Hysteretic $I-V$ curves of superconducting nanowires. Phys. Rev. B 68, 134515 (2003).

15. Li, P. et al. Retrapping current, self-heating, and hysteretic current-voltage characteristics in ultranarrow superconducting aluminum nanowires. Phys. Rev. B 84, 184508 (2011).

16. Glover, R. E. \& Sherrill, M. D. Changes in superconducting critical temperature produced by electrostatic charging. Phys. Rev. Lett. 5, 248-250 (1960).

17. Choi, J. et al. Electrical modulation of superconducting critical temperature in liquid-gated thin niobium films. Appl. Phys. Lett. 105, 012601 (2014).

18. Piatti, E. et al. Control of bulk superconductivity in a BCS superconductor by surface charge doping via electrochemical gating. Phys. Rev. B 95, 140501 (2017).

19. Zhao, Q.-Y. et al. A compact superconducting nanowire memory element operated by nanowire cryotrons. Supercond. Sci. Technol. 31, 035009 (2018).

20. Bezryadin, A. Superconductivity in Nanowires: Fabrication and Quantum Transport (Wiley-VCH, 2012).

21. Pracht, U. S. et al. Direct observation of the superconducting gap in a thin film of titanium nitride using terahertz spectroscopy. Phys. Rev. B $\mathbf{8 6}$ 184503 (2012)

22. Eisenmenger, W. \& Dayem, A. H. Quantum generation and detection of incoherent phonons in superconductors. Phys. Rev. Lett. 18, 125-127 (1967)
23. Dayem, A. H., Miller, B. I. \& Wiegand, J. J. Phonon generation and detection in superconducting lead diodes. Phys. Rev. B 3, 2949-2961 (1971).

24. Singer, I. L. \& Bron, W. E. Generation and detection of high-energy phonons by superconducting junctions. Phys. Rev. B 14, 2832-2841 (1976).

25. Ioffe, L. B., Geshkenbein, V. B., Helm, C. \& Blatter, G. Decoherence in superconducting quantum bits by phonon radiation. Phys. Rev. Lett. 93, 057001 (2004)

26. Brunetti, R. et al. Diffusion coefficient of electrons in silicon. J. Appl. Phys. 52, 6713-6722 (1981)

27. Fischetti, M. V., Laux, S. E. \& Crabbé, E. Understanding hot-electron transpor in silicon devices: is there a shortcut? J. Appl. Phys. 78, 1058-1087 (1995).

28. Sadasivam, S., Chan, M. K. Y. \& Darancet, P. Theory of thermal relaxation of electrons in semiconductors. Phys. Rev. Lett. 119, 136602 (2017).

29. Tanimura, H., Kanasaki, J., Tanimura, K., Sjakste, J. \& Vast, N. Ultrafast relaxation dynamics of highly excited hot electrons in silicon. Phys. Rev. B 100, 035201 (2019).

30. Sinha, S., Pop, E., Dutton, R. W. \& Goodson, K. E. Non-equilibrium phonon distributions in sub- $100 \mathrm{~nm}$ silicon transistors. J. Heat Transf. 128, 638-647 (2005)

31. Pop, E., Sinha, S. \& Goodson, K. Heat generation and transport in nanometer-scale transistors. Proc. IEEE 94, 1587-1601 (2006).

32. Ju, Y. S. \& Goodson, K. E. Phonon scattering in silicon films with thickness of order $100 \mathrm{~nm}$. Appl. Phys. Lett. 74, 3005-3007 (1999).

33. Anufriev, R., Ordonez-Miranda, J. \& Nomura, M. Measurement of the phonon mean free path spectrum in silicon membranes at different temperatures using arrays of nanoslits. Phys. Rev. B 101, 115301 (2020).

34. Chen, G., Freeman, R., Zholud, A. \& Urazhdin, S. Observation of anomalous non-ohmic transport in current-driven nanostructures. Phys. Rev. X 10, 011064 (2020).

35. Chen, G. \& Urazhdin, S. Transport and relaxation of current-generated nonequilibrium phonons from nonlocal electronic measurements. Preprint at https://arxiv.org/abs/2107.04634 (2021).

36. Roukes, M. L., Freeman, M. R., Germain, R. S., Richardson, R. C. \& Ketchen M. B. Hot electrons and energy transport in metals at millikelvin temperatures. Phys. Rev. Lett. 55, 422-425 (1985).

37. Wellstood, F. C., Urbina, C. \& Clarke, J. Hot-electron effects in metals. Phys. Rev. B 49, 5942-5955 (1994).

38. Basset, J. et al. Gate-assisted phase fluctuations in all-metallic Josephson junctions. Phys. Rev. Research 3, 043169 (2021)

39. Lambe, J. \& McCarthy, S. L. Light emission from inelastic electron tunneling. Phys. Rev. Lett. 37, 923-925 (1976).

40. Gimzewski, J. K., Reihl, B., Coombs, J. H. \& Schlittler, R. R. Photon emission with the scanning tunneling microscope. Z. Pysik B-Condens. Matter $\mathbf{7 2}$, 497-501 (1988).

41. Uehara, Y., Kimura, Y., Ushioda, S. \& Takeuchi, K. Theory of visible light emission from scanning tunneling microscope. Jpn. J. Appl. Phys. 31, 2465-2469 (1992).

42. Parzefall, M. et al. Antenna-coupled photon emission from hexagonal boron nitride tunnel junctions. Nat. Nanotechnol. 10, 1058-1063 (2015).

43. Doderer, M. et al. Light emission from a waveguide integrated MOS tunnel junction. In Conference on Lasers and Electro-Optics FW3C.1 (Optica Publishing Group, 2019).

44. Bude, J., Sano, N. \& Yoshii, A. Hot-carrier luminescence in Si. Phys. Rev. B 45, 5848-5856 (1992).

45. Lacaita, A. L., Zappa, F., Bigliardi, S. \& Manfredi, M. On the bremsstrahlung origin of hot-carrier-induced photons in silicon devices. IEEE Trans. Electron Devices 40, 577-582 (1993).

46. Natarajan, C. M., Tanner, M. G. \& Hadfield, R. H. Superconducting nanowire single-photon detectors: physics and applications. Supercond. Sci. Technol. 25, 063001 (2012)

47. Walsh, E. D. et al. Josephson junction infrared single-photon detector. Science 372, 409-412 (2021)

48. Morpurgo, A. F., Klapwijk, T. M. \& van Wees, B. J. Hot electron tunable supercurrent. Appl. Phys. Lett. 72, 966-968 (1998).

49. McCaughan, A. N. \& Berggreen, K. K. Superconducting three-terminal device and logic gates. US patent 9,509,315 B2 (2016).

50. McCaughan, A. N. et al. A superconducting thermal switch with ultrahigh impedance for interfacing superconductors to semiconductors. Nat. Electron. 2, 451-456 (2019).

51. Wagner, A., Ranzani, L., Ribeill, G. \& Ohki, T. A. Demonstration of a superconducting nanowire microwave switch. Appl. Phys. Lett. 115, 172602 (2020).

\section{Acknowledgements}

We are grateful to A. Pushp, B. Madon and M. A. Mueed for the deposition of TiN films and V. Geshkenbein for fruitful discussions. We thank the Cleanroom Operations Team of the Binnig and Rohrer Nanotechnology Center (BRNC) for their help and support. F.N. acknowledges support from the European Research Commission (grant no. 804273). A.F. acknowledges support from the Swiss National Science Foundation via grant no. 200021_188752. 


\section{Author contributions}

A.F. and F.N. conceived the experiments. M.F.R. and A.F. designed and fabricated the samples. N.C. and A.F. performed the simulations of the electric-field magnitude. M.F.R., M.H. and F.N. performed the measurements. D.Z.H. and C.B. fitted the switching probability data. All the authors analysed and interpreted the data and contributed to the writing of the manuscript.

\section{Competing Interests}

The authors declare the following competing interest: US patent $11,165,429 \mathrm{~B} 2$ (operating a superconducting channel by electron injection).

\section{Additional information}

Supplementary information The online version contains supplementary material available at https://doi.org/10.1038/s41928-022-00721-1.

Correspondence and requests for materials should be addressed to A. Fuhrer or F. Nichele.
Peer review information Nature Electronics thanks Loren Alegria, Yuri Pashkin and Alfredo Levy Yeyati for their contribution to the peer review of this work.

Reprints and permissions information is available at www.nature.com/reprints.

Publisher's note Springer Nature remains neutral with regard to jurisdictional claims in published maps and institutional affiliations.

\section{(c) (i)}

Open Access This article is licensed under a Creative Commons

Attribution 4.0 International License, which permits use, sharing, adaptation, distribution and reproduction in any medium or format, as long as you give appropriate credit to the original author(s) and the source, provide a link to the Creative Commons license, and indicate if changes were made. The images or other third party material in this article are included in the article's Creative Commons license, unless indicated otherwise in a credit line to the material. If material is not included in the article's Creative Commons license and your intended use is not permitted by statutory regulation or exceeds the permitted use, you will need to obtain permission directly from the copyright holder. To view a copy of this license, visit http://creativecommons. org/licenses/by/4.0/.

(c) The Author(s) 2022 